\title{
The origins of the mean-variance approach in finance: revisiting de Finetti 65 years later
}

\author{
Flavio Pressacco · Paolo Serafini
}

Received: 28 November 2006 / Accepted: 23 January 2007 - (C) Springer-Verlag 2007

\begin{abstract}
In a recent critical review of de Finetti's paper "Il problema dei pieni', the Nobel Prize winner Harry Markowitz recognized the primacy of de Finetti in applying the mean-variance approach to finance, but pointed out that de Finetti did not solve the problem for the general case of correlated risks. We argue in this paper that a more fair sentence would be: de Finetti did solve the general problem but under an implicit hypothesis of regularity which is not always satisfied. Moreover, a natural extension of de Finetti's procedure to non-regular cases offers a general solution for the correlation case and shows that de Finetti anticipated a modern mathematical programming approach to mean-variance problems.
\end{abstract}

Mathematics Subject Classification (2000): 91B30, 90C20

Journal of Economic Literature Classification: G11, C61, B23, D81, G22

\section{Introduction}

In a paper which appeared in 1940 (de Finetti (1940)) and which concerned proportional reinsurance decisions, Bruno de Finetti applied the mean-variance approach to solve a financial problem under uncertainty. The paper written in Italian in an actuarial journal and at the beginning of the Second World War went unnoticed by researchers in financial economics and knowledge of this work remained for a very long time restricted to European actuarial circles.

In the following decade, Markowitz published his milestone papers on the mean-variance approach to portfolio selection, which brought him the Nobel

\footnotetext{
F. Pressacco $(\varangle)$

Dipartimento di Finanza dell'Impresa e dei Mercati Finanziari, Università di Udine

P. Serafini

Dipartimento di Matematica e Informatica, Università di Udine
} 
Prize in Economics and the name of founder of modern finance (Markowitz $(1952,1956))$. In contrast de Finetti's paper fell into oblivion; he himself did not include it in a list of his own papers bearing some, even minor, connection with economic problems (de Finetti (1969)).

Only recently, alerted by second generation (or indirect) de Finetti scholars, leading economists of the English-speaking world recognized the importance of de Finetti's paper. In particular Rubinstein (2006a) expressed the opinion that de Finetti anticipated much of mean-variance portfolio theory and had quietly laid the foundations of modern finance theory and practice twelve years earlier than Markowitz. Markowitz (2006) himself, prompted by Rubinstein, offered a kind critical review of de Finetti's paper.

Among other considerations Markowitz found a shortcoming in de Finetti's treatment of the general case of correlated risks. It turns out that the procedure suggested by de Finetti (as a plain extension of the one correctly applied to the no-correlation case) to solve the problem under correlation among risks works only under a special hypothesis (which we call the regularity hypothesis) which may not be satisfied. Hence Markowitz concluded that de Finetti did not solve the general problem of correlated risks and also that advanced mathematical programming techniques, not yet known at the time of de Finetti's paper, must be applied to solve the proportional reinsurance problem in the general case. In turn Rubinstein (2006b) wrote that de Finetti 'fully worked out only an algorithm for solving the problem in the special case of uncorrelated returns'.

However, in our opinion these sentences, although literally correct, are too strong. It is our opinion that, as we show in this paper, the tools proposed by de Finetti, along with his insightful ideas, deserve and should receive a double re-evaluation. On the one hand a natural adjustment of his sequential procedure offers an easy solution to the general proportional reinsurance problem even in the case where the regularity hypothesis is not satisfied. On the other hand these tools may be successfully embedded in the modern environment of mathematical programming to pave the way for a meaningful sequential procedure to define the optimum path.

Quite likely de Finetti neglected the lack of regularity, thinking that it was an unlikely circumstance in the reinsurance market. To this aim, we also discuss conditions providing regularity in case of constant positive correlation among risks.

The paper is organized as follows. Section 2 recalls the essentials of de Finetti's approach to the mean-variance approach to optimum proportional reinsurance decisions. Section 3 describes de Finetti's correct treatment of the case of no correlation. Section 4 is devoted to the extension of the procedure to the case of correlated risks and to a discussion of the shortcomings of the regularity hypothesis implicit in de Finetti's treatment. Section 5 offers a correct 
extension of de Finetti's procedure to find the optimum path in the general correlation case. Sections 6 and 7 show how to exploit de Finetti's ideas in the current mathematical programming scenario. In Section 8 we provide an enlightening example of a three-dimensional non-regular case. Sufficient conditions for regularity are given in Section 9. In Section 10 we briefly discuss the connections with portfolio theory and CAPM in actuarial applications. A final conclusion is offered in Section 11. A technical appendix devoted to proving the results of Section 9 concludes the paper.

Before entering into the core of the paper we discuss our choice of symbols and terminology. As to the symbols we choose to adopt the ones typical in the optimization literature, but supplying a conversion table so as to allow the reader a better comparison with the papers of de Finetti (1940) and Markowitz (2006). As to the terminology, a more detailed explanation of our approach is in order. We tried to be as close as possible to de Finetti's style by making substantial recourse to geometric ideas. Indeed it was typical of de Finetti to use a geometric approach to analyze problems, to describe the steps leading logically to the solution and to explain the meaning and properties of the solution. This style also strongly characterizes his 1940 paper. It may be perceived today as unusual, but we think that it is a simple and powerful approach and at the same time a tribute to de Finetti's spirit.

\section{The pillars of de Finetti's approach}

We briefly recall the essentials of de Finetti's approach. An insurance company is faced with $n$ risks (policies). The net profit of these risks is represented by a vector of random variables with expected value $m=\left\{m_{i}>\right.$ $0 \quad i=1, \ldots, n\}$ and with a non-singular covariance matrix $\mathbf{V}= \begin{cases}\sigma_{i j} & i,\end{cases}$ $j=1, \ldots, n\}$. The company has to choose a proportional reinsurance or retention strategy specified by a retention vector $x$. The retention strategy is feasible if $0 \leq x_{i} \leq 1$ for all $i$. A retention $x$ induces a random profit with expected value $E=x^{\top} m$ and variance $V=x^{\top} \mathbf{V} x$.

A retention $x$ is by definition optimal (Pareto efficient) if, for no feasible retention $y$, we have both $x^{\top} m \leq y^{\top} m$ and $x^{\top} \mathbf{V} x \geq y^{\top} \mathbf{V} y$ with at least one inequality strict. Let $X^{*}$ be the set of optimal retentions. The core of de Finetti's approach is represented by the following simple and clever ideas, where geometric intuition plays an important role.

The set of feasible retentions is represented by points of the $n$-dimensional unit cube. The set $X^{*}$ is a path in this cube. It connects the natural starting point, the vertex 1 of full retention (with the largest expectation $E=\sum_{i} m_{i}$ ), to the opposite vertex $\mathbf{0}$ of full reinsurance (zero retention and hence minimum null variance). 
Consider a point $x^{*} \in X^{*}$ (with $x^{*}>\mathbf{0}$ ). Then de Finetti argues that a measure of the advantage coming from a (small) additional (or initial) reinsurance of the $i$-th risk is given by the value that the functions (defined on the unit cube for $x>\mathbf{0}$ in de Finetti (1940) and on the whole unit cube $x \geq \mathbf{0}$ in the extension of his method)

$$
F_{i}(x)=\frac{1}{2} \frac{\frac{\partial V}{\partial x_{i}}}{\frac{\partial E}{\partial x_{i}}}=\sum_{j=1}^{n} \frac{\sigma_{i j}}{m_{i}} x_{j}, \quad i=1, \ldots, n,
$$

assume at $x^{*}$. Note that $F_{i}(x)$ may be seen as (one half) the decrease of a properly normalized variance, i.e., the decrease of the variance measured in units of the expectation numeraire.

As we see below, the functions $F_{i}(x)$ play a key role in describing the efficient set. Taking this into account as well as the fact that the symbol used by de Finetti was $K_{i}$, we call these functions the key functions of our problem. The connection between the functions $F_{i}(x)$ and the optimal set $X^{*}$ comes from intuitive golden rules embedded in de Finetti's approach.

The first golden rule is that at any $x \in X^{*}$ an additional (or initial) reinsurance must be provided only for the $\operatorname{risk}(\mathrm{s}) i$ for which $F_{i}(x)$ is maximum. Intuitively, an additional reinsurance is only for those risks giving the biggest advantage. We write $\lambda(x)=\max _{i} F_{i}(x)$.

As we proceed along the optimum path, de Finetti argues that $\lambda(x) X^{*} \rightarrow$ $R$ is a monotonically decreasing continuous function, so that there is a one-toone correspondence between $x \in X^{*}$ and $\lambda(\mathbf{1}) \geq \lambda(x) \geq \lambda(\mathbf{0})$. The notation used by de Finetti for our $\lambda$ is $A$. As we see below, the parameter $\lambda$ is nothing but the counterpart of the Lagrange multiplier $\lambda$ in constrained optimization as well as in Markowitz (2006). Given its importance in determining the optimal set $X^{*}$, we call $\lambda$ the key parameter.

Consider the set $I(x)$ of those risks $i$ such that $F_{i}(x)=\lambda(x)$. If $I(x)$ is a singleton the direction of movement of the optimum path leaving $x$ is unequivocal. What if $I(x)$ has two or more elements? In such a case de Finetti provides a second golden rule.

Second golden rule: if $x \in X^{*}$ is a point for which $I(x)$ has more than one element, then leave $x$ in the direction in which all risks of $I(x)$ are additionally (or initially) reinsured in such a way as to preserve the equality of the $F_{i}(x)$ functions for all $i \in I(x)$.

Given the form of the $F_{i}(x)$ functions, this implies a movement on a segment of the cube characterized by the set of equations $F_{i}(x)=\lambda(x)$ for all $i \in$ $I(x)$. Note that only the risks belonging to $I(x)$ play an active role in defining the direction of the optimum path at $x$. They may be expressively named directional risks. Note also that in that direction the other non-directional risks are fixed at a constant retention level. Moreover, $F_{i}(x)<\lambda(x)$ for all $i \notin I(x)$, 
and, as long as the inequality is preserved, the direction does not change and the set $I(x)$ is invariant.

But, argues de Finetti, going in that direction along the optimum path, we will find a point $x$ (or a value $\lambda(x)$ ) such that one of the non-directional risks, say $j$, attains a value of $F_{j}(x)$ matching the common value $F_{i}(x)=\lambda(x)$ of all the previous directional risks and the corresponding inequality $\left(F_{j}(x)<\lambda(x)\right)$ is no longer preserved.

This introduces the third golden rule: at that point $x$ there is a change of direction in the optimum path. Indeed, also the risk $j$ is able to obtain at $x$, by a reinsurance movement, the greatest advantage $\lambda(x)$ so that it becomes coherently a member of the set $I(x)$ by joining the other current members. A directional change is then forced by the fact that, according to the second golden rule, a new set of equations $F_{i}(x)=\lambda(x)$, with the addition of the equation for the newcomer directional risk, determines the new direction of the optimum path.

We underline the need to be cautious in accepting the procedure just described. As we shall see, the procedure is correct in the correlation case only under a regularity condition to be introduced and discussed below and which de Finetti took granted in any case.

Although the procedure does not extend in its simple form to the general case, the role played by the key functions and the key parameter remains, as we shall see, fundamental in determining the optimum path. This shows the far-reaching intuition of de Finetti in a time when modern powerful mathematical programming techniques were not available. Moreover, de Finetti solved the problem in an algorithmic way, unusual at the time of his writing, when computers did not yet exist. We stress that it is the algorithmic nature of his method which allows for a simple computation of optimal points for any value of the expected profit even in the general case. We may say that he was a precursor of the celebrated critical line algorithm (CLA) of Markowitz (1956).

Before proceeding we find it useful to provide the reader with a correspondence table between the symbols used in this paper and the ones actually employed by de Finetti (1940) and Markowitz (2006). 


\begin{tabular}{|c|c|c|c|}
\hline & de Finetti & Markowitz & this paper \\
\hline retentions & $a_{i}$ & $X_{i}$ & $x_{i}$ \\
\hline key parameter & $A$ & $\lambda$ & $\lambda$ \\
\hline risk exp. values & $\bar{k}_{i}$ & $\mu_{i}$ or $m_{i}$ & $m_{i}$ \\
\hline risk cov. matrix & & $C$ & $\mathbf{V}$ \\
\hline risk cov. matrix entries & $r_{i j} \bar{\sigma}_{i} \bar{\sigma}_{j}$ & $\sigma_{i j}$ & $\sigma_{i j}$ \\
\hline key function & $K_{i}(a)$ & & $F_{i}(x)$ \\
\hline risk state & & $\mathrm{UP}$ & $I^{1}$ \\
\hline risk state & & $\mathrm{DOWN}$ & $I^{0}$ \\
\hline risk state & & $\mathrm{IN}$ & $I(x)$ and $I^{*}$ \\
\hline
\end{tabular}

\section{The no-correlation case}

We briefly describe in some detail how the procedure devised by de Finetti works in the simple case of no correlation, when $F_{i}(x)$ reduces to $x_{i} \sigma_{i i} / m_{i}$. We write $v_{i}=\sigma_{i i} / m_{i}$ (the normalized variance). Then $F_{i}(x)=F_{i}\left(x_{i}\right)=$ $x_{i} v_{i}$. It is convenient to order the risks according to the normalized variance values $v_{i}=F_{i}(1)$. Henceforth we suppose that there is a strict ordering assumption denoted the non-degenerate hypothesis, so that $v_{1}>v_{2}>\ldots>v_{n}$.

The correspondence between points $x \in X^{*}$, the key parameter $\lambda$ and the key functions $F_{i}(x)$ is captured by the following sequential procedure.

We start from the vertex $\mathbf{1}$. According to the first golden rule, we look for $\max F_{i}(\mathbf{1})=\max _{i} v_{i}=v_{1}$. The risk labelled 1 is the first to be reinsured. Accordingly $\lambda(\mathbf{1})=v_{1}=\max F_{i}(\mathbf{1})$. Then de Finetti points out that, for any value $0=\lambda(\mathbf{0}) \leq \lambda \leq \lambda(\mathbf{1})$ of the parameter $\lambda$, an optimal retention $x \in X^{*}$ is given by

$$
x_{i}=\min \left\{\lambda / v_{i}, 1\right\}, \quad i=1, \ldots, n .
$$

This means that, if $\lambda<v_{i}$, then $x_{i}=\lambda / v_{i}$ so that $F_{i}(x)=x_{i} v_{i}=\lambda$, while, if $\lambda>v_{i}$, then $x_{i}=1$ so that $F_{i}(x)=v_{i}<\lambda$. Let $v_{h}<\lambda<v_{h-1}$ for $h=2, \ldots, n+1$. Consistent with the second golden rule, the first $h-1$ risks obtain the biggest advantage $\lambda$ at any $x$ of the segment (or at any $\lambda$ of the interval) and play the role of directional risks along the segment identified by the equations $F_{i}(x)=\lambda, i=1, \ldots, h-1$, so that $I(x)=\{1, \ldots, h-1\}=$ $I^{h-1}$, while the other risks are frozen fully insured at the level 1 and do not belong to the set $I(x)$.

We now check what happens at $\lambda=v_{h}, h \leq n$. It is clear that here $x_{h}=$ $\lambda / v_{h}$ and also $F_{h}(x)=x_{h} v_{h}=\lambda$ while still $F_{i}(x)=x_{i} v_{i}=\lambda=v_{h}$ for 
$i=1, \ldots, h-1$, and $F_{i}(x)=v_{i}<\lambda=v_{h}$ for $i=h+1, \ldots, n$. But this means exactly that, at the value $\lambda=v_{h}$ and at the corresponding optimal point $x$, the third golden rule plays its role. There the matching occurs so that risk $h$ (responsible for the matching) enters into the set of directional risks, joining the other $h-1$ previously belonging to $I^{h-1}$. This implies that risk $h$ begins to be reinsured and stops being frozen at the level 1 of full retention. We call $\lambda^{h}=v_{h}$ a matching value and the corresponding $x^{h} \in X^{*}$ a matching point.

Summing up, for any matching value $\lambda^{h}$, we have $\lambda^{h}=F_{h}\left(x^{h}\right)=1 v_{h}$ (and so $\lambda^{1}=\lambda(\mathbf{1})=v_{1}$ ), while in general we have the following one-to-one correspondence between $x \in X^{*}$ and $\lambda^{1} \geq \lambda \geq 0$ :

$$
x_{i}=\min \left\{\lambda / v_{i}, 1\right\}, \quad i=1, \ldots, n, \quad \lambda=\max _{i} x_{i} v_{i} .
$$

Geometrically, this means that $X^{*}$ is described in the $n$-dimensional unit cube by a piecewise linear path, whose corner points $x^{h}$ are found jointly with the corresponding values of the key parameter $\lambda^{h}$. The corner point $x^{h}$ lies along the optimum direction given by the first $h-1$ risks, at the point where the common value of their key functions is matched from below by the key function of risk $h$ (while the other key functions have smaller values). In turn $\lambda^{h}$ is the common value of the key functions involved. Assuming the nondegenerate hypothesis, we find that $X^{*}$ consists of $n$ segments (otherwise it consists of a number of segments equal to the number of different $v_{i}$ values).

In Markowitz (2006) terminology the risks stay in one of three "states", according to the retention level: UP if the retention level is 1, DOWN if it is 0 and IN if it is strictly between 0 and 1 . In the no-correlation case the state transitions occur at each corner where risk $h$ changes state from UP to IN. At the final corner point $\mathbf{0}$ all risks jointly collapse to the retention 0 and change state from IN to DOWN.

\section{The general case of correlated risks in de Finetti's treatment}

In his treatment of the general case with correlated risks de Finetti suggests that the properties of the optimum path fully mimic the pattern characterizing the no-correlation case. The only difference is that we lack an a priori ordering of the risks because of a more complicated expression of the key functions

$$
F_{i}(x)=\sum_{j=1}^{n} \frac{\sigma_{i j}}{m_{i}} x_{j}, \quad i=1, \ldots, n,
$$

replacing $F_{i}(x)=x_{i} \sigma_{i i} / m_{i}$. Once again, we begin by defining $\lambda^{1}=\max _{i}$ $F_{i}(\mathbf{1})$. Let $k$ be the risk providing the largest value and $I^{1}=\{k\}$. Then a sequence of corner values $\lambda^{h}$, corner points $x^{h}$ and sets $I^{h}$ of directional risks 
is recursively found for $h=2, \ldots, n$. The direction of movement on $X^{*}$ for $\lambda^{h}<\lambda \leq \lambda^{h-1}$ is described by the equations

$$
\lambda=F_{i}(x), \quad i \in I^{h-1}, \quad x_{i}=1, \quad i \notin I^{h-1},
$$

which define $x$ parameterized by $\lambda$. The corner point $x^{h}$ is still found in this direction, where one of the non-directional risks (previously frozen at the level 1 of full retention) matches from below the common value of the key functions of the $h-1$ directional risks in $I^{h-1}$. The corner value $\lambda^{h}$ is defined as the smallest $\lambda^{h}$ such that

$$
F_{i}(x(\lambda)) \leq \lambda, \quad i \notin I^{h-1} .
$$

There, the new matching risk, providing the equality in (2), joins the set $I^{h-1}$ of the previous directional risks, changing state from UP to IN, and defining the new set $I^{h}$.

As before, all corner points are of the same matching type and the set $X^{*}$ is a piecewise linear path consisting of $n$ segments (under a non-degeneracy assumption that the equality in (2) is due only to one risk) in a one-to-one correspondence with the values of $\lambda$. Once again, all risks collapse jointly to the retention 0 .

Hence it could be said that the general case with correlation differs from that of no correlation only for the computational effort required to find $X^{*}$ through a truly sequential procedure, whereas in the no-correlation case the elegance and simplicity of the closed formulas (1) allows for an immediate computation.

We remark that de Finetti's approach takes for granted that at any stage a matching event occurs, that is, a feasible corner point is found along the current optimum direction. It can be shown by counterexamples, and was pointed out by Markowitz, that this does not always happen. Nevertheless, we can say that de Finetti worked with an implicit regularity assumption and gave a correct procedure to find the solution to the problem with correlated risks under this hypothesis of regularity. We repeat that the hypothesis consists in the existence of a feasible corner point, induced by a matching event, at any stage of his procedure. What if the assumption is not satisfied? We treat the point in the next section.

\section{An extension of de Finetti's procedure to the general case}

We start from the question left open at the end of the previous section. It is clear that regularity is violated if and only if along the optimum path one of the currently directional risks reaches a boundary value of the retentions before a matching event occurs. A boundary value is typically 0 , even if 1 cannot 
a priori be excluded once we accept that monotonicity of the retentions as functions of $\lambda$ is no longer available under correlation.

Consider a point $x^{*}$, or the corresponding value $\lambda^{*}$, where non-regularity occurs for the first time along the optimum path. We call such a point critical. This means that previously the conditions of regularity held. Then, without loss of generality, there were just $h-1$ previous directional risks for which $F_{i}(x)=\lambda$, while for the other non-directional risks, still at the level 1 of full retention, $F_{i}(x)<\lambda$. As a matching event on this current direction has not yet occurred, these relations still hold at the critical point $x^{*}$ (or critical value $\left.\lambda^{*}\right)$.

At this critical point the golden rules of de Finetti's procedure no longer hold. Indeed, while at this point the risks maximizing $F_{i}\left(x^{*}\right)$ are the current directional ones, we cannot move away from $x^{*}$ along this direction, as dictated by the first and second golden rules. Indeed advancing means going out of the unit cube because the critical risk becomes less than 0 or bigger than 1 .

We are then faced with the need of defining a new procedure to leave $x^{*}$ or, more generally, to deal with new situations not found in the regular case. As we already said, de Finetti did not treat the point, but, in our opinion, his tools (the key functions) and his logic (the golden rules), once properly adjusted, may nevertheless be successfully applied. In this section we present, in some detail, but informally and making large recourse to geometric intuitions, the adjusted procedure for the non-regular case.

First note that the critical point $x^{*}$ is surely a corner point of the optimum path because a compulsory change of direction occurs there. But this corner point is of a different type with respect to the ones found in the regular case. Those were driven by matching events. This was driven by what can be called a boundary event. As the ordinary rule to leave $x^{*}$ (or any critical point generated by a boundary event) cannot hold, we suggest, in the spirit of de Finetti's approach, following a new rule, as much as possible consistent with the ordinary golden rules of maximization of the advantage.

In order to find a new direction in as close a way as possible to the old logic, the only possibility is that one of the old directional risks leaves the previous directional set $I$, so that we are left with a set $I\left(x^{*}\right) \subset I$ of $h-2$ directional risks (provided $I\left(x^{*}\right)$ is not empty; this case is discussed below). Or, more formally, the new optimum direction from $x^{*}$ is obtained by the solution of the set of $h-2$ equations $F_{i}(x)=\lambda$ for $i \in I\left(x^{*}\right)$, together with $x_{i}=1$, for $i \notin I$. It remains to choose the risk to be excluded from $I$ to obtain $I\left(x^{*}\right)$. Since we need one more equation to determine the new direction, it seems quite obvious (at least in case the boundary value of the critical risk is 0 ) that this must be just the critical risk, responsible for the boundary event, and that the $n$-th equation is just setting this variable to 0 or to 1 , i.e, fixed to its boundary value. 
Applying this adjustment of the golden rules, we find a nice symmetry between the rules holding at a corner point of matching type and boundary type respectively. In the former case the critical (matching) risk joins, while in the latter the critical (boundary) risk leaves, the set of the previous directional risks. Now it is clear how to leave any type of corner point $x^{*}$ (unless $I\left(x^{*}\right)$ is empty).

Let us discuss what happens to the excluded critical risk along the newly defined optimum path. If it is excluded at a level 1 , of course its exclusion must be temporary. While it is waiting to regain an active role as a critical risk of a matching event for lower value of $\lambda$, it is obvious that in the meantime its key function must satisfy $F_{i}(x)<\lambda$, consistent with the rule holding in the regular case for the risks not yet entered in reinsurance.

If a critical risk is excluded at a level 0 , the temptation would be, at first glance, to consider this as a final exclusion, and to neglect the risk with no need to keep further into account the corresponding $F_{i}(x)$. Maybe surprisingly, this would be incorrect. As shown in the example in Section 8, the exclusion at a level 0 may be temporary as well. What happens in the meantime (or definitely if this is the case) to the excluded risk? Its key function must satisfy $F_{i}(x)>\lambda$. Indeed the exclusion is definitive if and only if, for all remaining points of the optimum path, $F_{i}(x)>\lambda$ for the critical risk $i$. And it is otherwise temporary up to the first $x$ where $F_{i}(x)=\lambda$.

A comment is in order to explain the logic behind these statements. Clearly, for a risk $i$ at the retention level 0 there is no intuitive reinsurance interpretation of $F_{i}(x)$. We cannot have an additional reinsurance for this risk. But, in a portfolio of reinsurance movements under correlation of risks, risks currently at 0 may also play a role. For risks of this type, coming back from full reinsurance surely increases the variance but also the expectation and we should look at the global effect of the combination with reinsurance movements of the other risks. Suppose, ad absurdum, that $F_{i}(x)<\lambda$. Then a small step back from full reinsurance combined with reinsurance movements of the other directional risks would open the way for a diminution of the global normalized variance greater than $\lambda$, a logical contradiction. And when $F_{i}(x)=\lambda$ for a lower value $\lambda$ of the parameter, this is just the point to come back from full reinsurance realizing a matching event of an unusual type. This is a matching event where the critical matching risk is not coming from level 1 as in the regular case, but from level 0 and the corresponding key function now matches from above, and not from below, the key functions of the current directional risks.

It is obvious that this adjusted procedure should be followed for any sequence of critical matching and boundary points. Before summarizing the results, we discuss the case in which, at a corner point of the boundary type, the set $I\left(x^{*}\right)$ is empty. In this case the adjusted procedure cannot immediately 
help us in escaping from the critical point $x^{*}$. Indeed there are no available directional risks. Note that such a point corresponds to a vertex of the unit cube as $x_{i}^{*} \in\{0,1\}$ for any $i$ as a consequence of the vanishing of the directional risks and in particular the critical risk is surely of type 0 to avoid logical contradictions.

Consider the current value of $\lambda$ at $x^{*}=x^{h}$. We label $\lambda$ as $\bar{\lambda}^{h}$, instead of simply as $\lambda^{h}$, for a reason which will be soon clear. According to our rules, for the set of risks at level 1 (surely non-empty as otherwise we would have reached the final vertex 0) $F_{i}\left(x^{*}\right)<\lambda$. For the set of risks at level 0, except for the critical one, $F_{i}\left(x^{*}\right)>\lambda$. And of course $F_{i}\left(x^{*}\right)=\lambda$ for the critical one at level 0 . Now we must adjust the procedure by decreasing $\lambda$; note that, in the absence of directional risks, the key functions all stay fixed at their level $F_{i}\left(x^{*}\right)$, so that decreasing $\lambda$ has no effect until the decreasing parameter $\lambda$ matches the biggest value among the $F_{i}\left(x^{*}\right)$ of the risks at level 1 . This is to be viewed as nothing but a new matching event. Accordingly the matching risk becomes the new singleton directional risk and things go on.

We may view this as a resetting of the procedure. Indeed the situation at this vertex looks like the one at the initial starting point, vertex $\mathbf{1}$, where we looked for $\max _{i} F_{i}(\mathbf{1})$ over the risks at level 1, i.e., among all risks. Here on the contrary we look for $\max _{i} F_{i}\left(x^{*}\right)$ only (once again) on those risks $i$ currently at level 1 and define $\underline{\lambda}^{h}$ as that maximum. In both cases the maximizing risk is the first to be reinsured in the path starting from the vertex. It may be said that a boundary point of this vertex type reveals a stronger non-regularity than the one characterizing other non-vertex boundary points. Indeed in the first case only, the one-to-one correspondence between points of $X^{*}$ and values of $0 \leq$ $\lambda \leq 1$ is lost: the critical vertex $x^{h}$ is associated to an interval $\bar{\lambda}^{h} \geq \lambda \geq \underline{\lambda}^{h}$.

Let us summarize the main results.

a) The procedure starts with the search for $\max _{i} F_{i}(\mathbf{1})$ which defines the first risk to be reinsured.

b) The optimum path is a piecewise linear path with possibly more than $n$ segments. The corner points of the path play a key role. They may be generated by two types of events: matching events and boundary events.

c) In a matching event the key function of a critical matching risk, whose retention value is previously frozen at a fixed level ( 1 or 0$)$, matches from below (for critical risks at level 1) or from above (for critical risks at level 0 ) the common value of the key function of the current directional risks, whose retention value was along the previous segment variable. When a matching event occurs, the matching risk (whatever its previous level 1 or 0 ) joins the set of previous directional risks. Its retention value starts changing, that is, it begins to be reinsured if the previous level was 1 , and on the contrary comes back from full reinsurance if its previous level was 
0 . The other risks do not change their state (directional or not) at the corner point.

d) In a boundary event the variable retention level of a risk belonging to the set of current directional risks reaches a boundary value (that is, the retention 0 or 1) before a matching event happens. When a boundary event occurs, the boundary risk leaves the set of directional risks. Its retention level becomes fixed at the boundary level reached. The other risks do not change their states at the corner point.

e) In case the set of remaining directional risks is not empty the way of leaving a corner point is the same for both types of corner points: move along the direction given by the set of equations $F_{i}(x)=\lambda$ for all $i$ in the new set of directional risks (the other non-directional risks being fixed at their respective levels). For internal points of each segment of the optimum path, besides having $F_{i}(x)=\lambda$ for all $i$ in the set of directional risks we also have $F_{i}(x)<\lambda$ for all $i$ in the set of risks fixed at the level 1 and $F_{i}(x)>\lambda$ for all risks fixed at level 0 .

f) In case the set of remaining directional risks is empty, the corner point is a vertex of the unit cube. The one-to-one correspondence between $x \in X^{*}$ and $\lambda$ is lost and, in order to leave the vertex, we need to decrease $\lambda$ to the smallest value of the interval associated to the vertex, where one of the risks at level 1 realizes a new matching event.

Markowitz (2006) puts into evidence that there are four types of corner according to the changes of state of the critical risk: a) from UP to IN, b) from IN to DOWN, c) from IN to UP, d) from DOWN to IN. By using this terminology our regularity condition corresponds to allowing transitions of type a) alone. We may also characterize matching corners as transitions of type a) or d) and boundary corners as transitions of type b) or c).

A complex hypothesis of non-degeneracy is implicitly assumed throughout the paper. It may be resumed by the condition that, at any value of the parameter $\lambda$ where a transition of state occurs, only one risk is in transition.

It is interesting to note that Markowitz qualifies the "backward transitions" (from IN to UP and from DOWN to IN) as unusual in de Finetti's model (Markowitz (2006), p. 14). He mentions them more in the form of a dubitative warning. He says that the possibilities of a risk $i$ moving from DOWN to IN or from IN to UP "may seem like unusual events in the de Finetti model; but we have no theorem that says they cannot happen, so the algorithm should be prepared to handle them'. In particular he exhibits a two-dimensional example in which the case DOWN-IN occurs but with a negative expectation of the risk involved. We provide in Section 8 a three-dimensional example with a standard positive expectation for all risks, where a DOWN-IN transition occurs. This reveals that there is no monotonic property of the reinsurance retentions along the optimum path as taken for granted in the proportional 
reinsurance problem. Note also that the presence of backward transitions is a sufficient but not necessary condition for rejecting monotonicity of the retentions.

A further terminological remark is in order, when we deal with monotonicity. We often used a dynamic point of view in our discussion. This is not intended in a temporal sense. For example when we speak of further reinsurance starting from $x$ we do not intend that a further reinsurance treaty is added to a previous already signed one. Also when we speak of coming back from full reinsurance we do not mean to retract part of a reinsurance treaty already signed. This is simply to understand the effect of modifying the reinsurance conditions holding at $x$. Of course it may well happen that an insurance company, after having concluded a treaty, may decide to arrange further reinsurance, perhaps because a new, more risk averse manager has been appointed. And in this case a new problem, with initial situation the one reached through the first treaty, should be analyzed, implying monotonicity at least with respect to the final position coming from the first treaty. But this has nothing to do with the problem studied by de Finetti.

\section{A mathematical programming formulation}

We now analyze the problem using mathematical programming techniques and show how the tools introduced by de Finetti, such as key functions and the key parameter, play a meaningful role and are the most natural way to build up a computational procedure to solve the optimum retention problem. This can be restated as the following quadratic problem:

$$
\begin{aligned}
\min & \frac{1}{2} x^{T} \mathbf{V} x \\
& m^{T} x=E \\
& \mathbf{0} \leq x \leq \mathbf{1}
\end{aligned}
$$

for every attainable $E$, i.e., $0 \leq E \leq \sum_{i} m_{i}$. The strict convexity of the objective function guarantees that there is a one-to-one correspondence between points in $X^{*}$ and optimal solutions of (3) for all attainable $E$. The KarushKuhn-Tucker conditions are necessary and sufficient for optimality of (3), since the constraints are regular and the objective function is strictly convex (see Shapiro (1979), Karush (1939), Kuhn and Tucker (1951)). The conditions are expressed through the Lagrangean function

$$
L(x, \lambda, u, v)=\frac{1}{2} x^{T} \mathbf{V} x+\lambda\left(E-m^{T} x\right)+u(x-\mathbf{1})-v x
$$


and state that $\hat{x}$ is optimal if and only if there exist Lagrange multipliers $(\hat{\lambda}, \hat{u}, \hat{v}), \hat{u} \geq 0, \hat{v} \geq 0$, such that:

1) $\hat{x}$ minimizes $L(x, \hat{\lambda}, \hat{u}, \hat{v})$,

2) $\hat{x}$ is feasible in (3),

3) either $\hat{x}_{j}=0 \quad$ or $\quad \hat{v}_{j}=0 \quad$ (or both) and either $\hat{x}_{j}=1 \quad$ or $\quad \hat{u}_{j}=0 \quad$ (or both).

According to the third condition, called complementarity, we can see that $0<$ $x_{j}<1$ implies $v_{j}=0$ and $u_{j}=0$. It is convenient to introduce a new variable defined as $w=u-v$ and unconstrained in sign. Then the complementarity condition is equivalent to:

$$
\begin{array}{rll}
x_{j}=0 & =>\quad w_{j} \leq 0, \\
0<x_{j}<1 & =>\quad w_{j}=0, \\
x_{j}=1 & =>\quad w_{j} \geq 0 .
\end{array}
$$

In order to verify 1) of (4), since $x$ is unconstrained (in the Lagrangean minimization), it is enough to compute

$$
\frac{\partial L}{\partial x}=\mathbf{V} x-\lambda m+u-v=\mathbf{V} x-\lambda m+w=0,
$$

i.e., componentwise

$$
\sum_{j} \sigma_{i j} x_{j}-\lambda m_{i}+w_{i}=0, \quad i=1, \ldots, n,
$$

or, equivalently,

$$
\sum_{j} \frac{\sigma_{i j}}{m_{i}} x_{j}+\frac{w_{i}}{m_{i}}=\lambda, \quad i=1, \ldots, n,
$$

so that, by using the key functions, we need to solve

$$
F_{i}(x)+\frac{w_{i}}{m_{i}}=\lambda, \quad i=1, \ldots, n .
$$

It is convenient to partition the policies into three sets (possibly empty) as

$$
I^{0}=\left\{\begin{array}{ll}
j & x_{j}=0
\end{array}, I^{1}=\left\{\begin{array}{ll}
j & x_{j}=1
\end{array}\right\}, I^{*}=\left\{\begin{array}{lll}
j & 0<x_{j}<1
\end{array}\right\} .\right.
$$

For notational simplicity, we do not indicate that these subsets actually depend on $x$.

Then the complementarity condition can be restated through the key functions in the following simple form: 
Optimality condition. $x$ is optimal if and only if there exists $\lambda$ such that

$$
F_{i}(x)=\lambda, i \in I^{*}, \quad F_{i}(x) \geq \lambda, i \in I^{0}, \quad F_{i}(x) \leq \lambda, i \in I^{1} .
$$

In case of uncorrelated risks (5) simplifies

$$
\frac{\sigma_{i i}}{m_{i}} x_{i}=\lambda, i \in I^{*}, \quad 0 \geq \lambda \quad \text { if } \quad I^{0} \neq \emptyset, \quad \frac{\sigma_{i i}}{m_{i}} \leq \lambda, i \in I^{1},
$$

which implies that $I^{0}=\emptyset$ for $E>0$. In this case de Finetti's analysis is always correct.

We now investigate the relation between $E$ and $\lambda$. It is a known fact that the optimal value of (3), viewed as a function of $E$, is a non-decreasing convex function (see Shapiro (1979)). Indeed, let $f(E)$ be the optimal value of (3) as a function of $E$. Let $x$ and $x^{\prime}$ be optima in (3) with right-hand sides $E$ and $E^{\prime}$ respectively and let $0<\alpha<1$. Then, by definition,

$$
\alpha f(E)+(1-\alpha) f\left(E^{\prime}\right)=\frac{1}{2}\left(\alpha x^{T} \mathbf{V} x+(1-\alpha) x^{\prime T} \mathbf{V} x^{\prime}\right) .
$$

Moreover,

$$
m^{T}\left(\alpha x+(1-\alpha) x^{\prime}\right)=\alpha E+(1-\alpha) E^{\prime}
$$

so that the solution $\left(\alpha x+(1-\alpha) x^{\prime}\right)$ is feasible (but not necessarily optimal) in (3) with right-hand side $\left(\alpha E+(1-\alpha) E^{\prime}\right)$. Then

$$
\begin{aligned}
f\left(\alpha E+(1-\alpha) E^{\prime}\right) & \leq \frac{1}{2}\left(\alpha x+(1-\alpha) x^{\prime}\right)^{T} \mathbf{V}\left(\alpha x+(1-\alpha) x^{\prime}\right) \\
& <\frac{1}{2}\left(\alpha x^{T} \mathbf{V} x+(1-\alpha) x^{\prime T} \mathbf{V} x^{\prime}\right)
\end{aligned}
$$

where the second inequality follows from the strict convexity of the quadratic form. By combining this inequality and (6) we get the strict convexity of $f(E)$. Moreover, the function $f(E)$ is strictly increasing. Indeed, let $x$ be the optimum with right-hand side $E$ and consider the solution $\alpha x$ with $0<$ $\alpha<1$, so that $\alpha x$ is feasible with respect to the constraints $0 \leq x \leq 1$ and the constraint $\alpha m^{T} x=\alpha E$. Then

$$
f(E)=\frac{1}{2} x^{T} \mathbf{V} x>\frac{1}{2}(\alpha x)^{T} \mathbf{V}(\alpha x) \geq f(\alpha E),
$$

where the first inequality derives from the positive definiteness of the quadratic form together with $0 \leq \alpha<1$ and the second from the fact that $f(\alpha E)$ is the optimal value.

It is a known fact that the Lagrange multiplier $\lambda$ is a subgradient of $f(E)$ and is in fact its derivative if $f(E)$ is differentiable at $E$ (Shapiro (1979)). Hence $\lambda>0$ for any $E>0$ and also $\lambda$ is a strictly increasing function of $E$ (by strict convexity), although not necessarily continuous.

This implies that we may vary indirectly $E$ by varying $\lambda$ in (5) instead. This justifies the previous introduction of $\lambda$ as a key parameter. 


\section{A computational procedure}

The conditions of Section 6 suggest the following extension of de Finetti's approach to the general case: the starting point is the optimum $x=\mathbf{1}$ (and therefore $E=m^{T} \mathbf{1}, I^{0}=I^{*}=\emptyset$ ). Then (5) is:

$$
F_{i}(\mathbf{1}) \leq \lambda \quad \forall i \quad=>\quad \lambda=\max _{i} F_{i}(\mathbf{1})
$$

This is the largest value for $\lambda$, corresponding to $E=m^{T} \mathbf{1}$. Let $k$ be an index for which the maximum in (7) is attained. Then we update the sets $I^{1}$ and $I^{*}$ by moving $k$ from $I^{1}$ to $I^{*}$.

Then we recursively carry out the following computations until $\lambda=0$. If $I^{*} \neq \emptyset$, the optimal $x$ as a function of $\lambda$, given by $F_{i}(x)=\lambda, i \in I^{*}$, is computed by first solving the following two linear systems (in the variables $y$ and $z$ respectively)

$$
\sum_{j \in I^{*}} \frac{\sigma_{i, j}}{m_{i}} y_{j}=1, i \in I^{*}, \text { and } \sum_{j \in I^{*}} \frac{\sigma_{i, j}}{m_{i}} z_{j}=\sum_{j \in I^{1}} \frac{\sigma_{i, j}}{m_{i}}, i \in I^{*},
$$

and then combining

$$
x_{j}=\lambda y_{j}-z_{j}, \quad j \in I^{*} .
$$

Now we check which condition is violated first by decreasing $\lambda$,

$$
F_{i}(x) \geq \lambda, i \in I^{0}, \quad 0 \leq x_{i} \leq 1, i \in I^{*}, \quad F_{i}(x) \leq \lambda, i \in I^{1},
$$

and then recompute the sets $I^{0}, I^{*}, I^{1}$. For the sake of notational simplicity, for $i \in I^{0}$, let

$$
\psi_{i}=\sum_{j \in I^{*}} \frac{\sigma_{i, j}}{m_{i}} y_{j}, \quad \phi_{i}=\sum_{j \in I^{*}} \frac{\sigma_{i, j}}{m_{i}} z_{j}, \quad \rho_{i}=\sum_{j \in I^{1}} \frac{\sigma_{i, j}}{m_{i}} .
$$

Then the conditions are checked by using (9) as follows: for $i \in I^{0}$, we have

$$
F_{i}(x(\lambda))=\sum_{j \in I^{*}} \frac{\sigma_{i, j}}{m_{i}}\left(\lambda y_{j}-z_{j}\right)+\sum_{j \in I^{1}} \frac{\sigma_{i, j}}{m_{i}}=\psi_{i} \lambda-\phi_{i}+\rho_{i} \geq \lambda
$$

from which

$$
\left(\psi_{i}-1\right) \lambda \geq \phi_{i}-\rho_{i}
$$

and so the value of $\lambda$ for which $F_{i}(x)=\lambda, i \in I^{0}$, is given by

$$
\lambda_{0}=\max \left\{\frac{\phi_{i}-\rho_{i}}{\psi_{i}-1} \quad i \in I^{0}, \psi_{i}-1>0\right\} .
$$

Similarly, for $i \in I^{1}$, we have

$$
\left(1-\psi_{i}\right) \lambda \geq \rho_{i}-\phi_{i}
$$


and so the value of $\lambda$ for which $F_{i}(x)=\lambda, i \in I^{1}$, is given by

$$
\lambda_{1}=\max \left\{\frac{\phi_{i}-\rho_{i}}{\psi_{i}-1} \quad i \in I^{1}, \psi_{i}-1<0\right\} .
$$

To check $0 \leq x_{i} \leq 1$ we simply compute as follows:

$$
\begin{aligned}
\lambda y_{i}-z_{i}=0 & =>\quad \lambda_{* 0}=\max \left\{\frac{z_{i}}{y_{i}} \quad i \in I^{*}, y_{i}>0\right\}, \\
\lambda y_{i}-z_{i}=1 & =>\quad \lambda_{* 1}=\max \left\{\frac{1+z_{i}}{y_{i}} \quad i \in I^{*}, y_{i}<0\right\} .
\end{aligned}
$$

Then the corner value $\lambda^{h}$ is given by

$$
\lambda^{h}=\max \left\{\lambda_{0}, \lambda_{1}, \lambda_{* 0}, \lambda_{* 1}\right\}
$$

and the corner points $x^{h}$ are given by $x_{j}^{h}=\lambda^{h} y_{j}-z_{j}, j \in I^{*}, x_{j}^{h}=0, j \in I^{0}$, $x_{j}^{h}=1, j \in I^{1}$.

We assume that there is no degeneracy in the data. In other words we assume that all maxima in the above expressions are achieved by a unique index. Let $k$ be this index. The sets are recomputed as follows: if $\lambda^{h}=\lambda_{0}$ then $k$ moves from $I^{0}$ to $I^{*}$; if $\lambda^{h}=\lambda_{1}$ then $k$ moves from $I^{1}$ to $I^{*}$; if $\lambda^{h}=\lambda_{* 0}$ then $k$ moves from $I^{*}$ to $I^{0}$; if $\lambda^{h}=\lambda_{* 1}$ then $k$ moves from $I^{*}$ to $I^{1}$. In the first two cases $x^{h}$ is a matching point and in the other two cases $x^{h}$ is a boundary point.

If $I^{*}=\emptyset$ (and necessarily $x$ is a vertex of the unit cube), then (5) simplifies to $F_{i}(x) \geq \lambda, i \in I^{0}, F_{i}(x) \leq \lambda, i \in I^{1}$, from which we only have to recompute $\lambda=\max \left\{F_{i}(x) \quad i \in I^{1}\right\}$ (with the discontinuity already mentioned). Note that this implies that, whenever $I^{*}=\emptyset$, the index entering $I^{*}$ comes from $I^{1}$. As previously remarked, the interval

$$
\min \left\{F_{i}\left(x^{h}\right) \quad i \in I^{0}\right\} \geq \lambda \geq \max \left\{F_{i}\left(x^{h}\right) \quad i \in I^{1}\right\}
$$

is associated to the same vertex corner point $x^{h}$. For the two special corner values, extremes of the interval (11), we adopt the notation:

$$
\bar{\lambda}^{h}=\min \left\{F_{i}\left(x^{h}\right) \quad i \in I^{0}\right\}, \quad \underline{\lambda}^{h}=\max \left\{F_{i}\left(x^{h}\right) i \in I^{1}\right\} .
$$

We briefly comment on the possibility that the index $k$ providing the maximum is not unique. This means that the violation of the complementarity is simultaneously due to different conditions for the same value of $\lambda$ (so-called degeneracy). We may take as $k$ any index providing the maximum and continue the procedure as if it were unique. It may happen that there is a sequence of steps such that the sets $I^{0}, I^{*}, I^{1}$ change at each step while $\lambda$ remains constant. This is similar to the degeneracy of the simplex steps in linear programming (see Dantzig (1963)) and therefore we should take care of possible 
cycling of the method, in the sense that the same pattern of changes of the three sets is repeated for ever while $\lambda$ remains constant. We do not dwell on this technical problem here.

\section{A critical example}

We now consider a case with three risks whose expected profit and covariance matrix are

$$
m=\left(\begin{array}{l}
1 \\
1 \\
1
\end{array}\right), \mathbf{V}=\frac{1}{100}\left(\begin{array}{rrr}
160 & 96 & 24 \\
96 & 90 & 27 \\
24 & 27 & 40
\end{array}\right),
$$

so the key functions are

$$
\begin{aligned}
& F_{1}(x)=\frac{1}{1000}\left(160 x_{1}+96 x_{2}+24 x_{3}\right), \\
& F_{2}(x)=\frac{1}{1000}\left(96 x_{1}+90 x_{2}+27 x_{3}\right), \\
& F_{3}(x)=\frac{1}{1000}\left(24 x_{1}+27 x_{2}+40 x_{3}\right) .
\end{aligned}
$$

For $E=\sum_{j} m_{j}=3$, the optimal retention is $x=\mathbf{1}$. The key function values at the starting point $\mathbf{1}$ are

$$
F_{1}(\mathbf{1})=\frac{280}{1000}=\frac{7}{25}, \quad F_{2}(\mathbf{1})=\frac{213}{1000}, \quad F_{3}(\mathbf{1})=\frac{91}{1000} .
$$

The condition

$$
\lambda(\mathbf{1})=\lambda^{1}=\max _{i} F_{i}(\mathbf{1})
$$

gives

$$
\lambda^{1}=\frac{7}{25}, \quad I^{*}=\{1\}, \quad I^{1}=\{2,3\}, \quad I^{0}=\emptyset .
$$

Hence the risk 1 is a directional risk (the only one at the moment). The direction is given by $F_{1}\left(x_{1}, 1,1\right)=\lambda$ from which we get

$$
x_{1}=\frac{1000 \lambda-96-24}{160} \text {. }
$$

The conditions in (10) correspond to the inequalities:

$$
0 \leq x_{1} \leq 1=>0 \leq \frac{1000 \lambda-96-24}{160} \leq 1=>\frac{3}{25} \leq \lambda \leq \frac{7}{25},
$$




$$
\begin{aligned}
& F_{2}(x)=\frac{1}{1000}\left(96 \frac{1000 \lambda-96-24}{160}+90+27\right) \leq \lambda=>\lambda \geq \frac{9}{80}, \\
& F_{3}(x)=\frac{1}{1000}\left(24 \frac{1000 \lambda-96-24}{160}+27+40\right) \leq \lambda=>\lambda \geq \frac{49}{850} .
\end{aligned}
$$

To check which condition is violated first by decreasing $\lambda$ we compute

$$
\max \left\{\frac{3}{25} ; \frac{9}{80} ; \frac{49}{850}\right\}=\frac{3}{25} \text {. }
$$

This means that we have reached a boundary vertex corner $x^{2}=(0,1,1)$ and the sets must be changed to $I^{*}=\emptyset, I^{0}=\{1\}, I^{1}=\{2,3\}$. Then we have the special corner value $\bar{\lambda}^{2}=3 / 25$. At $x^{2}$ the key functions have values

$$
F_{1}(0,1,1)=\frac{120}{1000}=\frac{3}{25}, F_{2}(0,1,1)=\frac{117}{1000}, F_{3}(0,1,1)=\frac{67}{1000},
$$

and $\lambda$ is reset to the value

$$
\underline{\lambda}^{2}=\max \left\{\frac{117}{1000} ; \frac{67}{1000}\right\}=\frac{117}{1000} .
$$

Moving away from $x^{2}$ the index sets become $I^{0}=\{1\}, I^{*}=\{2\}, I^{1}=\{3\}$, with risk 2 being the directional risk. The direction is given by $F_{2}\left(0, x_{2}, 1\right)=$ $\lambda$, from which we get:

$$
\frac{1}{1000}\left(90 x_{2}+27\right)=\lambda \quad=>\quad x_{2}=\frac{1000 \lambda-27}{90} .
$$

Now the conditions in (10) correspond to the inequalities:

$$
\begin{gathered}
0 \leq x_{2} \leq 1=>\frac{27}{1000} \leq \lambda \leq \frac{117}{1000}, \\
F_{1}\left(0, x_{2}, 1\right)=\frac{1}{1000}\left(96 \frac{1000 \lambda-27}{90}+24\right) \geq \lambda=>\lambda \geq \frac{9}{125}, \\
F_{3}\left(0, x_{2}, 1\right)=\frac{1}{1000}\left(27 \frac{1000 \lambda-27}{90}+40\right) \leq \lambda=>\lambda \geq \frac{319}{7000} .
\end{gathered}
$$

Since

$$
\max \left\{\frac{27}{1000} ; \frac{9}{125} ; \frac{319}{7000}\right\}=\frac{9}{125},
$$

this means that we have reached a matching corner, $x^{3}=(0,1 / 2,1)$, with matching value $\lambda^{3}=9 / 125$. Moving away from $x^{3}$ the index sets become $I^{*}=\{1,2\}, I^{0}=\emptyset, I^{1}=\{3\}$, and we have two directional risks 1 (coming 
back from full retention) and 2. To find the direction we have to solve the system

$$
F_{1}\left(x_{1}, x_{2}, 1\right)=\lambda, \quad F_{2}\left(x_{1}, x_{2}, 1\right)=\lambda,
$$

i.e., from (8) and (9)

$$
\begin{gathered}
\frac{1}{1000}\left(\begin{array}{rr}
160 & 96 \\
96 & 90
\end{array}\right)\left(\begin{array}{l}
y_{1} \\
y_{2}
\end{array}\right)=\left(\begin{array}{l}
1 \\
1
\end{array}\right), \\
\frac{1}{1000}\left(\begin{array}{rr}
160 & 96 \\
96 & 90
\end{array}\right)\left(\begin{array}{l}
z_{1} \\
z_{2}
\end{array}\right)=\frac{1}{1000}\left(\begin{array}{l}
24 \\
27
\end{array}\right),
\end{gathered}
$$

from which

$$
\left(\begin{array}{l}
x_{1} \\
x_{2}
\end{array}\right)\left(\begin{array}{r}
-\frac{125}{108} \\
\frac{1000}{81}
\end{array}\right) \lambda-\left(\begin{array}{r}
-\frac{1}{12} \\
\frac{7}{18}
\end{array}\right),
$$

Now the conditions in (10) correspond to the inequalities:

$$
\begin{aligned}
& 0 \leq x_{1} \leq 1 \quad=>\quad-\frac{99}{125} \leq \lambda \leq \frac{9}{125}, \\
& 0 \leq x_{2} \leq 1 \quad=>\quad \frac{63}{2000} \leq \lambda \leq \frac{9}{80},
\end{aligned}
$$

$$
\begin{gathered}
F_{3}\left(x_{1}, x_{2}, 1\right)= \\
\frac{1}{1000}\left(24\left(-\frac{125}{108} \lambda+\frac{1}{12}\right)+27\left(\frac{1000}{81} \lambda-\frac{7}{12}\right)+40\right) \leq \lambda \\
=>\quad \lambda \geq \frac{567}{12500} .
\end{gathered}
$$

Since

$$
\max \left\{-\frac{99}{125} ; \frac{63}{2000} ; \frac{567}{12500}\right\}=\frac{567}{12500},
$$

we have reached a matching corner $x^{4}=(37 / 1200,77 / 450,1)$, with matching value $\lambda^{4}=567 / 12500$. In order to leave $x^{4}$ the index sets become $I^{*}=$ $\{1,2,3\}, I^{0}=I^{1}=\emptyset$. All risks are directional and the direction is given by the solution of $\mathbf{V} x=\lambda \mathbf{1}$, which provides the last segment of $X^{*}$. Thus,

$$
x=\left(\begin{array}{c}
\frac{4625}{6804} \\
\frac{2750}{729} \\
\frac{12500}{567}
\end{array}\right) \lambda .
$$

Finally for $\lambda=0$ we obtain the end point of $X^{*}$, i.e., the vertex $\mathbf{0}$. Summing up, the optimal $x_{i}$ and the optimal expected profit $E$ as functions of $\lambda$ are (note the non-monotonic behavior of $x_{1}$ ): 


\begin{tabular}{rrrrrr}
$\lambda$ & \multicolumn{1}{c}{$x_{1}$} & $x_{2}$ & $x_{3}$ & $E$ \\
$\frac{7}{25} \geq \lambda$ & $\geq \frac{3}{25}$ & $\frac{25}{4} \lambda-\frac{3}{4}$ & 1 & 1 & $\frac{25}{4} \lambda+\frac{5}{4}$ \\
$\frac{3}{25} \geq \lambda$ & $\geq \frac{117}{1000}$ & 0 & 1 & 1 & 3 \\
$\frac{117}{1000} \geq \lambda \geq \frac{9}{125}$ & 0 & $\frac{100}{9} \lambda-\frac{3}{10}$ & 1 & $\frac{100}{9} \lambda+\frac{7}{10}$ \\
$\frac{9}{125} \geq \lambda$ & $\geq \frac{567}{12500}$ & $-\frac{125}{108} \lambda+\frac{1}{12}$ & $\frac{1000}{81} \lambda-\frac{7}{18}$ & 1 & $\frac{3625}{324} \lambda+\frac{25}{36}$ \\
$\frac{567}{12500} \geq \lambda$ & $\geq 0$ & $\frac{4625}{6804} \lambda$ & $\frac{2750}{729} \lambda$ & $\frac{12500}{567} \lambda$ & $\frac{540875}{20412} \lambda$
\end{tabular}

In Figs. 1(a) and 1(b) we display the non-monotonic function $x_{1}(\lambda)$ and the set $X^{*}$ within the unit cube (note that $X^{*}$ consists of four segments, i.e., one more than the number of risks).

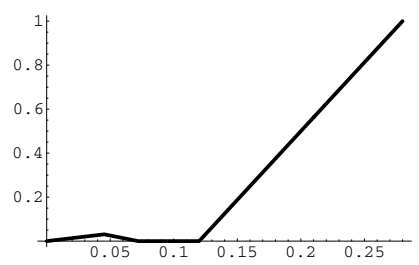

(a) $x_{1}(\lambda)$

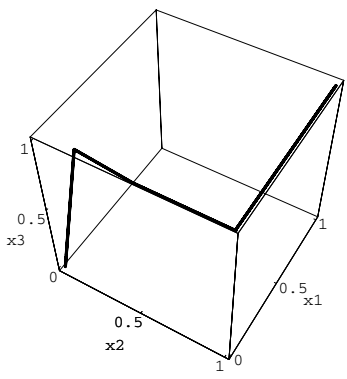

(b) $X^{*}$

Fig. 1

It is interesting to note that this is an example of a non-regular case in which the last segment is fully internal, that is, all variables jointly reach the value 0 (but not for the first time, at least for the risk 1). We also summarize the set of transitions involved:

\begin{tabular}{|c|c|c|c|}
\hline type of transition & risks involved & corner point $x^{h}$ & corner value $\lambda^{h}$ \\
\hline UP-IN & 1 & $(1,1,1)$ & $7 / 25$ \\
\hline IN-DOWN & 1 & $(0,1,1)$ & $3 / 25$ \\
\hline UP-IN & 2 & $(0,1,1)$ & $117 / 1000$ \\
\hline DOWN-IN & 1 & $(0,1 / 2,1)$ & $72 / 1000$ \\
\hline UP-IN & 3 & $(37 / 1200,77 / 450,1)$ & $567 / 125000$ \\
\hline IN-DOWN & $1,2,3$ & $(0,0,0)$ & 0 \\
\hline
\end{tabular}


This shows that the internality of the last segment is a necessary, but not a sufficient, condition for regularity. Hence it may be said that the problem of regularity goes far beyond the simple behavior of the last segment on which the criticism of Markowitz (2006), p. 6, regarding the so-called last segment conjecture, was mainly based.

\section{Conditions for regularity}

We restate here the concept of regularity for an optimum path.

Definition. The optimal path $X^{*}$ is regular if at corner points risks only move from $I^{1}$ to $I^{*}$.

In other words regularity implies that the violation of the complementarity conditions (5) is always referred to $F_{i}(x) \leq \lambda$. As already stated, no correlation among the risks guarantees regularity of the optimum path. However, regularity can obtain even if the risks are correlated provided certain conditions are met. In order to investigate these conditions it is convenient to rewrite $m$ as $m_{i}=\eta_{i} \sigma_{i}$ (with $\sigma_{i}=\sqrt{\sigma_{i i}}$ ), where each $\eta_{i}$ plays the role of a safety loading coefficient under a standard deviation premium principle. Then $m=\Sigma \eta$, with $\Sigma=\operatorname{diag}\left\{\sigma_{1}, \ldots, \sigma_{n}\right\}$. Moreover,

$$
\mathbf{R}=\Sigma^{-1} \mathbf{V} \Sigma^{-1},
$$

where $\mathbf{R}=\left\{r_{i j} i, j=1, \ldots, n\right\}$ is the correlation matrix, i.e.,

$$
r_{i j}=\left\{\begin{aligned}
1 & \text { if } i=j, \\
\frac{\sigma_{i j}}{\sigma_{i} \sigma_{j}} & \text { if } i \neq j .
\end{aligned}\right.
$$

It is possible to provide sufficient conditions for regularity under the following assumptions: $r_{i j}=r \geq 0$ for all $i \neq j$ in the correlation matrix $\mathbf{R}$ and $\eta_{i} / \eta_{j} \leq K$ for all $i, j$, where $K$ is a positive constant. For instance, if all $\eta_{i}$ are equal, then $K=1$. One preliminary result (proved in the Appendix) is given in the following proposition.

Proposition 1. If $r n(K-1)<1-r$, then no index $j \in I^{*}$ can move to $I^{1}$ by decreasing $\lambda$.

Note that in the two particular cases of all $\eta_{i}$ equal or $r=0$, the condition is satisfied. Our main result (proved in the Appendix) is as follows.

Proposition 2. If $3 n r(K-1)<1-r$, the optimum path $X^{*}$ is regular.

The bound provided in Proposition 2 is not tight. The various inequalities used to derive it are not tight and therefore the result is somehow pessimistic. 

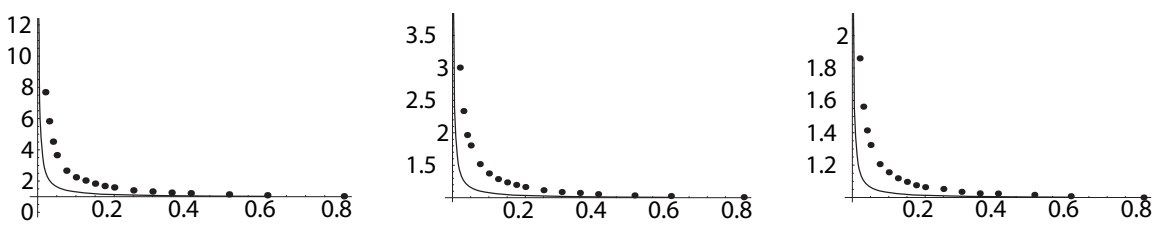

Fig. 2 Test results for $n=10, n=40$ and $n=100$
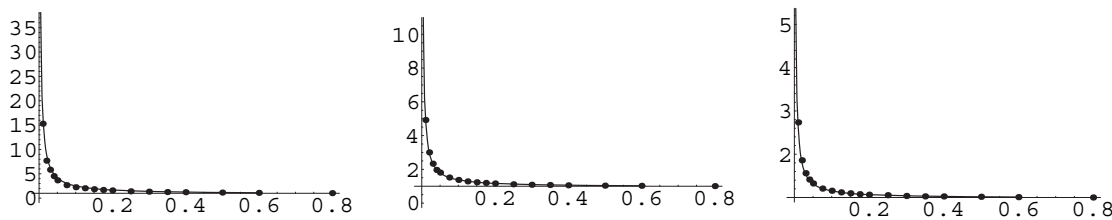

Fig. 3 Modified bound for $n=10, n=40$ and $n=100$

However, it does reveal a qualitative behavior of the relationship between $r$ and $K$ under which regularity holds. We carried out an experimental test for $n=10, n=40$ and $n=100$. We chose the following grid of values for $r$ :

$$
\begin{array}{r}
r \in 0.01,0.02,0.03,0.04,0.05,0.075,0.1,0.125,0.15 \\
0.175,0.2,0.25,0.3,0.35,0.4,0.5,0.6,0.8\}
\end{array}
$$

For each value of $r$ we found by binary search the maximum value of $K$ such that the following regularity test is successful: for each pair $(r, K)$ up to 100 random instances are generated and in each instance $\eta_{1}=1$ and $\eta_{2}=K$ while the values of $\eta_{i} \in 1, K$ for $i=3, \ldots, n$, and $\sigma_{i} \in 5,10$ for $i=1, \ldots, n$, are randomly chosen. If all 100 instances exhibit a regular behavior then the test for the pair $(r, K)$ is declared successful, otherwise the test for the pair $(r, K)$ is stopped at the non-regular instance and the test for the pair $(r, K)$ is declared unsuccessful. The binary search is carried out until the tolerance interval for $K$ has length 0.01 .

In Fig. 2 we show both the curves corresponding to the bound in Proposition 2, i.e., the equation $3 n r(K-1)=1-r$ for the values $n=10, n=40$, $n=100$, and also the points found by the binary search. As expected the bound is not tight, in the sense that there are values not satisfying the bound for which the procedure exhibited a regular behavior. However, the qualitative hyperbolic relation between $r$ and $K$ is reflected both in the bound and in the computed points.

If we multiply the bound by the constant 5 we find an interesting and rather curious match between the experimental points and the theoretical bound. Empirically we may therefore substitute the bound in Proposition 2 by $3 n r(K-$ $1)<5(1-r)$ (see the new curve and the experimental points in Fig. 3 ). 


\section{Connections with portfolio theory and CAPM}

We would also like to acknowledge a suggestion by Bühlmann (2006), a nonanonymous referee of our paper, which paved the way for us to offer an interesting alternative interpretation of the optimality conditions holding under regularity. With $G_{i}$ denoting the random profit coming from an integral retention of the $i$ th risk, the suggestion is that in case of no correlation the set of Pareto efficient retention quotas (seen as a function of the non-negative parameter $\lambda$ ) should satisfy, for each $i, V\left(x_{i} G_{i}\right) / E\left(x_{i} G_{i}\right) \leq \lambda$ with $x_{i}=1$ if the inequality holds in strict form. In case of correlation an analogous condition is suggested by replacing only the numerators of the left-hand sides of the (in)equalities with $\operatorname{cov}\left(x_{i} G_{i}, \sum x_{i} G_{i}\right)$. The conditions are clearly equivalent (as long as $x_{i}>0$ for all $i$, that is, except for the point $\mathbf{0}$ of full reinsurance of all risks) to those found by de Finetti. In particular, under correlation they hold only in the regular case.

Let us give here just a sketch of their actuarial meaning: once we fix a threshold level $\lambda$ of acceptable normalized risk (variance measured in units of the numeraire, the expectation), efficient proportional reinsurance strategies are those that, whenever needed, cut down through reinsurance the normalized risk of each policy at the threshold, while do not touch those already under the threshold. In case of no correlation the proper (before normalization) risk measure of each policy is its variance (more precisely, the variance of its random gain after reinsurance); in the correlation case it is (also after reinsurance) its covariance with a reference portfolio. For any given retention choice, the reference portfolio is simply the one defined by the retentions. That this measure of risk may play a role in case of correlation is no longer surprising, at least after the diffusion in the actuarial world, mainly due to the path-breaking papers Borch (1974) and Bühlmann and Gerber (1978), of the results of the capital asset pricing model (Sharpe (1964), Lintner (1965), Mossin (1966)).

In essence this key role comes from the fact that $V\left(\sum_{i} x_{i} G_{i}\right)=$ $\sum_{i} \operatorname{cov}\left(x_{i} G_{i}, \sum_{i} x_{i} G_{i}\right)$, which shows that the contribution of each policy (after reinsurance) to the global amount of risk retained (the global variance after reinsurance) is precisely $\operatorname{cov}\left(x_{i} G_{i}, \sum_{i} x_{i} G_{i}\right)$. We do not want to enter here in greater detail about this very interesting point, but we remark that an early discussion of connections between de Finetti's work and portfolio theory can be found in Pressacco (1986) as quoted in Rubinstein (2006a).

\section{Conclusions}

It is today plainly recognized that de Finetti introduced the mean-variance approach in financial problems under uncertainty and, treating a problem of proportional reinsurance, offered a correct procedure to find the mean-variance 
Pareto efficient set in case of no correlation. As far as de Finetti's contribution in the general case of correlation is concerned, this is still an open question. We show in this paper that de Finetti gave a fully correct procedure (a plain extension of that offered for the no-correlation case), able to solve the problem with correlated risks only under a convenient regularity hypothesis. Quite likely, convinced that in practical actuarial applications non-regularity could not be relevant, he did not discuss a procedure of general validity (also applicable in the non-regular case).

To fill this gap and to complete the picture, we offer here a natural adjustment of de Finetti's procedure, which, respecting his logic and applying his simple and original tools, provides a correct solution to finding the optimum set also in the case of non-regularity.

It is well-known that (as discussed in some detail in Markowitz (2006)) the mean-variance proportional reinsurance problem may also be solved through an application of modern constrained optimization techniques. We show as well that de Finetti's tools also play a meaningful role in this advanced frame and that they provide the most natural way to building up a computational procedure consistent with constrained optimization techniques.

We also discuss sufficient conditions for regularity to hold also in case of correlated risks.

\section{Appendix 1}

Here we provide the proofs of Propositions 1 and 2 previously stated.

Proof of Proposition 1. Suppose that for a value $\lambda$ there are sets $I^{0}=\emptyset, I^{*}$ and $I^{1}$ such that the optimality condition is satisfied. We partition the matrix $\mathbf{V}$ into submatrices $\mathbf{V}_{1}^{1}, \mathbf{V}_{*}^{1}, \mathbf{V}_{1}^{*}, \mathbf{V}_{*}^{*}$, and similarly the vectors $a, w$ and $x$ into subvectors $a_{1}, a_{*}, w_{1}, w_{*}, x_{1}$ and $x_{*}$ respectively, where the superscript refers to the column indices and the subscript to the row indices. Then the optimality condition can be rewritten as (note that we are assuming $I^{0}=\emptyset$ )

$$
\mathbf{V}_{*}^{*} x_{*}+\mathbf{V}_{*}^{1} \mathbf{1}=\lambda a_{*}, \quad \mathbf{V}_{1}^{*} x_{*}+\mathbf{V}_{1}^{1} \mathbf{1} \leq \lambda a_{1},
$$

and so

$$
x_{*}=\mathbf{V}_{*}^{*-1}\left(-\mathbf{V}_{*}^{1} \mathbf{1}+\lambda a_{*}\right) .
$$

Then the various conditions become:

$$
\begin{array}{lclc}
x_{i}^{*} \geq 0, & i \in I^{*} & \text { if } & \mathbf{V}_{*}^{*-1} \mathbf{V}_{*}^{1} \mathbf{1} \leq \lambda \mathbf{V}_{*}^{*-1} a_{*}, \\
x_{i}^{*} \leq 1, & i \in I^{*} & \text { if } \quad \lambda \mathbf{V}_{*}^{*-1} a_{*} \leq \mathbf{1}+\mathbf{V}_{*}^{*-1} \mathbf{V}_{*}^{1} \mathbf{1}, \\
F_{i}(x) \leq \lambda, & i \in I^{1} & \text { if } & -\mathbf{V}_{1}^{*} \mathbf{V}_{*}^{*-1} \mathbf{V}_{*}^{1} \mathbf{1}+\mathbf{V}_{1}^{1} \mathbf{1} \leq \\
& & & \lambda\left(a_{1}-\mathbf{V}_{1}^{*} \mathbf{V}_{*}^{*-1} a_{*}\right) .
\end{array}
$$


If all entries of $\mathbf{V}_{*}^{*-1} a_{*}$ are non-negative, then the condition $x_{i}^{*} \leq 1$ can never be violated by decreasing $\lambda$. So we first investigate the question of when $\mathbf{V}_{*}^{*-1} a_{*} \geq 0$. We rewrite $\mathbf{V}_{*}^{*}=\Sigma_{*}^{*} \mathbf{R}_{*}^{*} \Sigma_{*}^{*}, a^{*}=\Sigma_{*}^{*} \eta_{*}$ so that

$$
\mathbf{V}_{*}^{*-1} a_{*}=\Sigma_{*}^{*-1} \mathbf{R}_{*}^{*-1} \eta_{*}
$$

and $\Sigma_{*}^{*-1} \mathbf{R}_{*}^{*-1} \eta_{*}>0$ if and only if $\mathbf{R}_{*}^{*-1} \eta_{*}>0$.

So we have (with $m=\left|I^{*}\right|$ )

$$
\mathbf{R}_{*}^{*}=\left(\begin{array}{cccc}
1 & r & \ldots & r \\
r & 1 & \ldots & r \\
\ldots \ldots & \ldots & \ldots \\
r & r & \ldots & 1
\end{array}\right)
$$

and

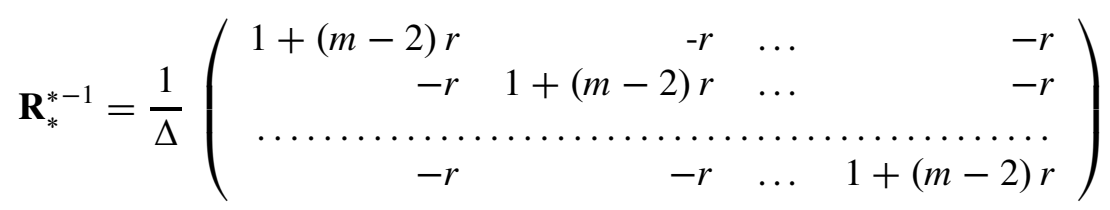

with

$$
\Delta=1+(m-2) r-(m-1) r^{2}=(1-r)(r(m-1)+1) .
$$

Then $\mathbf{R}_{*}^{*-1} \eta_{*}>0$ if and only if, for all $i \in I^{*}$,

$$
(1+(m-1) r) \eta_{i}-r \sum_{j \in I^{*}} \eta_{j}>0,
$$

i.e., if $r>0$,

$$
\frac{\eta_{i}}{\sum_{j \in I^{*}} \eta_{j}}>\frac{r}{1+(m-1) r}
$$

Since

$$
\frac{\eta_{i}}{\sum_{j \in I^{*}} \eta_{j}}=\frac{1}{\sum_{j \in I^{*}} \frac{\eta_{j}}{\eta_{i}}} \geq \frac{1}{m K},
$$

the condition is satisfied if

$$
\frac{1}{m K}>\frac{r}{1+(m-1) r}
$$

i.e.,

$$
1-r>r m(K-1),
$$

which in turn is satisfied if (since $n \geq m$ and $K \geq 1$ )

$$
1-r>r n(K-1) \text {. }
$$

Hence we may state the proposition in the following form. 
Proposition 1. If (13) holds, then no index $j \in I^{*}$ can move to $I^{1}$ by decreasing $\lambda$.

Proof of Proposition 2. Now we consider the condition $x^{*} \geq 0$. By using the expressions $\mathbf{V}_{*}^{*}=\Sigma_{*}^{*} \mathbf{R}_{*}^{*} \Sigma_{*}^{*}, a^{*}=\Sigma_{*}^{*} \eta_{*}, \mathbf{V}_{*}^{1}=\Sigma_{*}^{*} \mathbf{R}_{*}^{1} \Sigma_{1}^{1}$, the condition in (12) can be expressed as

$$
\Sigma_{*}^{*-1} \mathbf{R}_{*}^{*-1} \Sigma_{*}^{*-1} \Sigma_{*}^{*} \mathbf{R}_{*}^{1} \Sigma_{1}^{1} \mathbf{1} \leq \lambda \Sigma_{*}^{*-1} \mathbf{R}_{*}^{*-1} \Sigma_{*}^{*-1} \Sigma_{*}^{*} \eta_{*},
$$

i.e.,

$$
\Sigma_{*}^{*-1} \mathbf{R}_{*}^{*-1} \mathbf{R}_{*}^{1} \Sigma_{1}^{1} \mathbf{1} \leq \lambda \Sigma_{*}^{*-1} \mathbf{R}_{*}^{*-1} \eta_{*}
$$

equivalent to, since $\Sigma_{*}^{*}$ is a positive diagonal matrix,

$$
\mathbf{R}_{*}^{*-1} \mathbf{R}_{*}^{1} \Sigma_{1}^{1} \mathbf{1} \leq \lambda \mathbf{R}_{*}^{*-1} \eta_{*} .
$$

The condition $F_{i}(x) \leq \lambda$, after substituting $\mathbf{V}_{*}^{1}=\Sigma_{*}^{*} \mathbf{R}_{*}^{1} \Sigma_{1}^{1}, \mathbf{V}_{1}^{*}=$ $\Sigma_{1}^{1} \mathbf{R}_{1}^{*} \Sigma_{*}^{*}, \mathbf{V}_{1}^{1}=\Sigma_{1}^{1} \mathbf{R}_{1}^{1} \Sigma_{1}^{1}, \mathbf{V}_{*}^{*-1}=\Sigma_{*}^{*-1} \mathbf{R}_{*}^{*-1} \Sigma_{*}^{*-1}$ in (12), becomes

$$
-\Sigma_{1}^{1} \mathbf{R}_{1}^{*} \mathbf{R}_{*}^{*-1} \mathbf{R}_{*}^{1} \Sigma_{1}^{1} \mathbf{1}+\Sigma_{1}^{1} \mathbf{R}_{1}^{1} \Sigma_{1}^{1} \mathbf{1} \leq \lambda\left(\Sigma_{1}^{1} \eta_{1}-\Sigma_{1}^{1} \mathbf{R}_{1}^{*} \mathbf{R}_{*}^{*-1} \eta_{*}\right),
$$

i.e., since $\Sigma_{1}^{1}$ is positive diagonal,

$$
-\mathbf{R}_{1}^{*} \mathbf{R}_{*}^{*-1} \mathbf{R}_{*}^{1} \Sigma_{1}^{1} \mathbf{1}+\mathbf{R}_{1}^{1} \Sigma_{1}^{1} \mathbf{1} \leq \lambda\left(\eta_{1}-\mathbf{R}_{1}^{*} \mathbf{R}_{*}^{*-1} \eta_{*}\right) .
$$

Let $b=\mathbf{R}_{*}^{*-1} \mathbf{R}_{*}^{1} \Sigma_{1}^{1} \mathbf{1}, c=\mathbf{R}_{*}^{*-1} \eta_{*}, d=\mathbf{R}_{1}^{1} \Sigma_{1}^{1} \mathbf{1}$ so that (14) and (15) can be written as

$$
b \leq \lambda c, \quad-\mathbf{R}_{1}^{*} b+d \leq \lambda\left(\eta_{1}-\mathbf{R}_{1}^{*} c\right) .
$$

We can compute as follows:

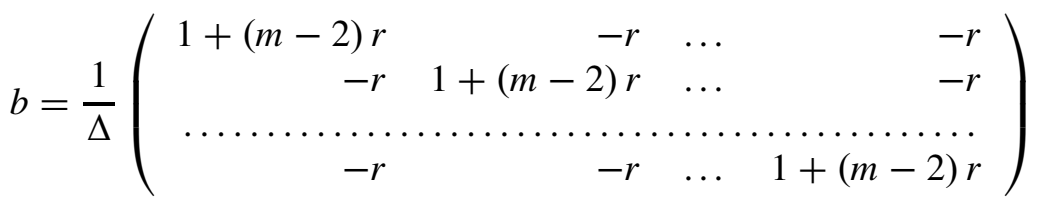

$$
\begin{aligned}
& \left(\begin{array}{cccc}
r & r & \ldots & r \\
r & r & \ldots & r \\
\cdots & \ldots & \ldots \\
r & r & \ldots & r
\end{array}\right) \Sigma_{1}^{1} \mathbf{1}=> \\
& b_{i}=\frac{r(1-r)}{\Delta} \sum_{j \in I^{1}} \sigma_{j}=\frac{r}{r(m-1)+1} \sum_{j \in I^{1}} \sigma_{j}, \quad i \in I^{*}, \\
& c_{i}=\frac{\eta_{i}(1+r(m-1))-r \sum_{j \in I^{*}} \eta_{j}}{\Delta}, \quad i \in I^{*} ;
\end{aligned}
$$




$$
\begin{aligned}
& d=\left(\begin{array}{cccc}
1 & r & \ldots & r \\
r & 1 & \ldots & r \\
\ldots & \ldots & \ldots & \ldots \\
r & r & \ldots & 1
\end{array}\right) \Sigma_{1}^{1} \mathbf{1}=>d_{i}=(1-r) \sigma_{i}+r \sum_{j \in I^{1}} \sigma_{j}, \quad i \in I^{1}, \\
& \left(d-\mathbf{R}_{1}^{*} b\right)_{i}=(1-r) \sigma_{i}+r \sum_{j \in I^{1}} \sigma_{j}-m r \frac{r}{r(m-1)+1} \sum_{j \in I^{1}} \sigma_{j} \\
& =(1-r) \sigma_{i}+\left(1-\frac{m r}{r(m-1)+1}\right) r \sum_{j \in I^{1}} \sigma_{j} \\
& =(1-r)\left(\sigma_{i}+\frac{r(1-r)}{r(m-1)+1} \sum_{j \in I^{1}} \sigma_{j}\right), \\
& \left(\eta_{1}-\mathbf{R}_{1}^{*} c\right)_{i}=\eta_{i}-r \frac{\sum_{j \in I^{*}} \eta_{i}(1+r(m-1))-m r \sum_{j \in I^{*}} \eta_{j}}{\Delta} \\
& =\eta_{i}-\frac{r}{r(m-1)+1} \sum_{j \in I^{*}} \eta_{i} .
\end{aligned}
$$

Then the first inequality in (16) becomes

$$
\frac{r(1-r)}{\Delta} \sum_{j \in I^{1}} \sigma_{j} \leq \lambda \frac{\eta_{i}(1+r(m-1))-r \sum_{j \in I^{*}} \eta_{j}}{\Delta}
$$

i.e.,

$$
r(1-r) \sum_{j \in I^{1}} \sigma_{j} \leq \lambda\left(\eta_{i}(1-r)+r\left(\eta_{i} m-\sum_{j \in I^{*}} \eta_{j}\right)\right), \quad i \in I^{*},
$$

and the second becomes

$$
(1-r) \sigma_{i}+\frac{(1-r)}{r(m-1)+1} r \sum_{j \in I^{1}} \sigma_{j} \leq \lambda\left(\eta_{i}-\frac{r}{r(m-1)+1} \sum_{j \in I^{*}} \eta_{i}\right),
$$
i.e.,

$$
\Delta \sigma_{i}+(1-r) r \sum_{j \in I^{1}} \sigma_{j} \leq \lambda\left(\eta_{i}(1-r)+r\left(\eta_{i} m-\sum_{j \in I^{*}} \eta_{j}\right)\right), \quad i \in I^{1} .
$$

Write $\gamma_{i}=\left(\eta_{i}(1-r)+r\left(\eta_{i} m-\sum_{j \in I^{*}} \eta_{j}\right)\right), \rho=(1-r) r \sum_{j \in I^{1}} \sigma_{j}$ so we have

$$
\lambda \geq \frac{\rho}{\gamma_{i}} \quad \text { for } \quad \gamma_{i}>0, \quad i \in I^{*},
$$




$$
\lambda \geq \frac{\Delta \sigma_{i}+\rho}{\gamma_{i}} \quad \text { for } \quad \gamma_{i}>0, \quad i \in I^{1} .
$$

For regularity to hold we must have

$$
\max \left\{\frac{\Delta \sigma_{i}+\rho}{\gamma_{i}} \gamma_{i}>0, \quad i \in I^{1}\right\}>\max \left\{\frac{\rho}{\gamma_{i}} \gamma_{i}>0, \quad i \in I^{*}\right\},
$$

or, equivalently,

$$
\exists i \in I^{1}, \gamma_{i}>0 \quad 1+\frac{\Delta \sigma_{i}}{\rho}>\frac{\gamma_{i}}{\gamma_{j}} \quad \forall j \in I^{*}, \gamma_{j}>0 .
$$

We first examine the question of the existence of $\gamma_{i}>0, i \in I^{1}$,

$$
\eta_{i}(1+r(m-1))-r \sum_{j \in I^{*}} \eta_{j}>0 .
$$

We have

$$
\begin{aligned}
& \eta_{i}(1+r(m-1))-r \sum_{j \in I^{*}} \eta_{j} \\
= & \eta_{i}\left(1+r(m-1)-r \sum_{j \in I^{*}} \frac{\eta_{j}}{\eta_{i}}\right)>\eta_{i}(1+r(m-1)-r m K) \\
= & \eta_{i}(1-r-r m(K-1))>\eta_{i}(1-r-r n(K-1)) .
\end{aligned}
$$

If (13) holds, then (18) is satisfied. We examine (17):

$$
\begin{aligned}
\frac{\gamma_{i}}{\gamma_{j}}-1 & =\frac{\eta_{i}(1-r)+r\left(\eta_{i} m-\sum_{k \in I^{*}} \eta_{k}\right)}{\eta_{j}(1-r)+r\left(\eta_{j} m-\sum_{k \in I^{*}} \eta_{k}\right)}-1 \\
& =\frac{\eta_{i}}{\eta_{j}} \frac{(1-r)+r\left(m-\sum_{k \in I^{*}} \frac{\eta_{k}}{\eta_{i}}\right)}{(1-r)+r\left(m-\sum_{k \in I^{*}} \frac{\eta_{k}}{\eta_{j}}\right)}-1 \\
& \leq K \frac{(1-r)+r m(1-1 / K)}{(1-r)+r m(1-K)}-1=\frac{(K-1)(1-r+2 r m)}{(1-r)-r m(K-1)} .
\end{aligned}
$$

Since there always exists an index $i \in I^{1}$ such that $\sigma_{i} \geq \sum_{j \in I^{1}} \sigma_{j} /(n-m)$ (in a set of numbers there is a number not less than the average), there exists $i \in I^{1}$ such that

$$
\frac{\Delta \sigma_{i}}{\rho}=\frac{(r(m-1)+1) \sigma_{i}}{r \sum_{j \in I^{1}} \sigma_{j}} \geq \frac{(1-r+r m)}{r(n-m)} .
$$

If

$$
\frac{(1-r+r m)}{r(n-m)}>\frac{(K-1)(1-r+2 r m)}{(1-r)-r m(K-1)}
$$


then (17) holds. Inequality (19) is equivalent to

$$
(K-1) r<\frac{(1-r+r m-r m K)(1-r+r m)}{(1-r+2 r m)(n-m)} .
$$

Since

$$
\frac{1-r+r m}{1-r+2 r m}>\frac{1}{2}
$$

and $1-r+r m-r m K \geq 1-r+r n-r n K$, we have

$$
\frac{(1-r+r m-r m K)(1-r+r m)}{(1-r+2 r m)(n-m)}>\frac{1}{2} \frac{(1-r+r n-r n K)}{n} .
$$

Then, if

$$
(K-1) r<\frac{1}{2} \frac{(1-r+r n-r n K)}{n},
$$

(19) and in turn (17) hold. A straightforward computation shows that (20) is equivalent to

$$
3 n r(K-1)<1-r .
$$

Note that (21) implies (13). So we may state the proposition as follows.

Proposition 2. If (21) holds, the optimum path $X^{*}$ is regular.

\section{References}

Borch, K. (1974): The mathematical theory of insurance. Lexington Books, Lexington, MA

Bühlmann, H., Gerber, H. (1978): Risk bearing and the reinsurance market. The ASTIN Bulletin 10, 12-24

Dantzig, G.B. (1963): Linear programming and extensions. Princeton University Press, Princeton, $\mathrm{NJ}$

de Finetti, B. (1940): Il problema dei “Pieni”. Giornale dell' Istituto Italiano degli Attuari 11, 1-88; translation (Barone, L. (2006)): The problem of full-risk insurances. Chapter I. The risk within a single accounting period. Journal of Investment Management 4(3), 19-43

de Finetti, B. (1969): Un matematico e l'economia. Franco Angeli, Milan

Karush, W. (1939): Minima of functions of several variables with inequalities as side conditions. S.M. dissertation. University of Chicago, Chicago, IL

Kuhn, H.W., Tucker, A.W. (1951): Nonlinear programming. In: Neyman, J. (ed.): Proceedings of the Second Berkeley Symposium on Mathematical Statistics and Probability. University of California Press, Berkeley, CA, pp. 481-492

Lintner, J. (1965): The valuation of risky assets and the selection of risky investments in stock portfolios and capital budgets. The Review of Economics and Statistics 47, 13-37

Markowitz, H. (1952): Portfolio selection. The Journal of Finance 7, 77-91

Markowitz, H. (1956): The optimization of a quadratic function subject to linear constraints. Naval Research Logistics Quarterly 3, 111-133 
Markowitz, H. (2006): de Finetti scoops Markowitz. Journal of Investment Management 4(3), 5-18

Mossin, J. (1966): Equilibrium in a capital asset market. Econometrica 34, 768-783

Pressacco, F. (1986): Separation theorems in proportional reinsurance. Goovaerts, M. et al. (eds.): Insurance and Risk Theory. D. Reidel, Dordrecht, pp. 209-215

Rubinstein M. (2006a ): Bruno de Finetti and mean-variance portfolio selection. Journal of Investment Management 4(3), 3-4

Rubinstein M. (2006b): A history of the theory of investments. Wiley, Hoboken, NJ

Shapiro, J.F. (1979): Mathematical programming: structures and algorithms. Wiley-Interscience, New York

Sharpe, W. (1964): Capital asset prices: a theory of market equilibrium under conditions of risk. The Journal of Finance 19, 425-442 